\title{
Fluorescence and photostability studies of anthracene-9-carboxylic acid in different media
}

\author{
M. S. A. Abdel-Mottaleb, ${ }^{1,2, \dagger}$ H. R. Galal, ${ }^{1}$ A. F. M. Dessouky, ${ }^{1}$ M. El-Naggar, ${ }^{1}$ \\ D. Mekkawi, ${ }^{1}$ S. S. Ali, ${ }^{2}$ and G. M. Attya ${ }^{2}$ \\ ${ }^{1}$ Photoenergy Center and \\ ${ }^{2}$ Department of Chemistry, Faculty of Science, Ain Shams University, 11566 Abbassia Cairo, Egypt
}

\begin{abstract}
Electronic absorption and fluorescence spectra of anthracene-9-carboxylic acid (ANCA) were studied in different homogeneous solvents, binary protic/aprotic solvent mixtures and in heterogeneous solutions of the cationic cetyltrimethyl ammonium bromide (CTAB) micelle. Different chemical species of ANCA were identified spectroscopically in different media. The results are discussed on the basis of a mechanism that involves two equilibria: acid-base equilibrium and monomer-dimer equilibrium. These equilibria were found to be very sensitive to the nature of the medium and the concentration of ANCA.

Moreover, while it is photostable in most solvents studied, the ANCA was found to be photolabile in aqueous media of different pHs. The acid-base catalyzed photodegradation rate was studied by following up absorption and/or fluorescence intensities as a function of illumination dose. The determined rate of the photochemical degradation of ANCA depends on the nature of the medium. The first order degradation rate constant is remarkably enhanced in heterogeneous medium of CTAB. As expected, the determined activation energy is low $\left(\sim 3.2 \mathrm{~kJ} . \mathrm{mol}^{-1}\right)$. This result favors photooxidation process. Anthraquinone was the main photodegradation product obtained via 9,9' -dicarboxylic head-to-head dimer of anthracene that was identified by GC-Mass technique.
\end{abstract}

\section{INTRODUCTION}

Recognition of events at the molecular level in chemistry, biology, and material science has become one of the most active and important research areas at present. Information about these events can be obtained via light signals. Applications of fluorescence probes and fluorescence-based molecular devices are getting so numerous that the desire to find new fluorophores and even to study old ones is becoming vital.

Anthracene and its derivatives constitute one class of the simple fluorophores that are widely employed as fluorescent sensors and in molecular switches [1, 2, 3, 4, 5, 6, 7, 8, 9, 10, 11, 12, 13, 14]. It has been found that the photophysics of anthracene9-carboxylic acid (ANCA) is quite different from that of anthracene. The fluorescence of ANCA exhibits a pronounced solvent and concentration dependence $[1,2,3,4,5,6,7]$. In water, the fluorescence spectrum is sharp and structured in a more anthracene like behavior. In ethanol however, an additional peculiar broad band around $470 \mathrm{~nm}$ is observed. The broad band<smiles>O=C(O)c1c2ccccc2cc2ccccc12</smiles>

around $470 \mathrm{~nm}$ was suggested to be arising from different equilibria; acid-base equilibrium [4],

\footnotetext{
${ }^{\dagger}$ Author to whom correspondence should be addressed
} E-mail: solar@photoenergy.org monomer-dimer equilibrium [2], or dimer-tetramer equilibrium [3].

Preliminary results obtained in our laboratories showed that this broad band prevails in aprotic solvents like acetone and cyclohexane and persists in micellar solutions. Moreover, in aqueous solutions of ANCA we have observed some degree of photolability. This prompted us to carry out an extensive study of the fluorescence and absorption spectra as well as photostability studies of ANCA in different homogeneous solvents and heterogeneous media of CTAB surfactant. In this paper we report and discuss the new results obtained in our laboratories for the chosen ANCA. A simple mechanism for the pathway of the photodegradation of ANCA will be also discussed.

\section{MATERIALS AND METHODS}

9-Anthracenecarboxylic acid (ANCA, Fluka, 98\%) was purified by recrystallization from ethanol. Cetyltrimethyl ammonium bromide (CTAB) from $\mathrm{BDH}$ was used as received. Surfactant solutions with ANCA were sonicated for several hours and allowed to stand overnight before measurements.

Absorption spectra were recorded with an UNICAM (He入ios- $\alpha$ ) spectrophotometer.

Fluorescence and excitation spectra were taken with a Schimadzu 5301PC spectrofluorophotometer.

The photochemical reactor (ACE GLASS INC.) is a cylindrical double walled quartz tube, $50 \mathrm{~mm}$ diameter, with inlet and outlet for cooling by water to maintain the temperature constant. 
During light irradiation the ANCA solutions were stirried with THERMOLYNE magnetic stirrer.

The light source used was 75 watt xenon arc lamp (PTI) powered with an PTI model LPS-250 arc lamp power supply.

The light intensity was measured using INTERNATIONAL LIGHT research radiometer model IL1700 equiped with appropriate solar-blind vacuum photodiodes head.

The $\mathrm{pH}$ of the solution was adjusted by addition of $\mathrm{NaOH}$ solution or $\mathrm{HClO}_{4}$ acid.

Fluorescence lifetime was measured using computer controlled Time Correlated Single Photon Counting Spectrometer FL900 from Edinburgh Instruments with hydrogen filled nF900 flash lamp. Using a $5 \times 10^{-5} \mathrm{M}$ concentration of degassed cyclohexane solution of anthracene sample of single decay time of $5.15 \mathrm{~ns}$ $( \pm 0.05 \mathrm{~ns})$ tested instrument performance. All solutions of ANCA were purged by dry Argon for few minutes before lifetime measurements.

\section{RESULTS AND DISCUSSION}

3.1. Photophysics of ANCA in homogeneous solutions. The absorption and fluorescence spectra of ANCA in different solvents are shown in Figures 1 and 2. Spectral data together with solvent parameters are summarized in Table 1. The absorption spectrum is slightly sensitive to the nature of the solvent compared to the fluorescence spectrum. The peaks at 384, 365, 347 , and $330 \mathrm{~nm}$ in the absorption spectrum of water solution of ANAC are assigned to the ${ }^{1} \mathrm{~L}_{\mathrm{a}}$ band.

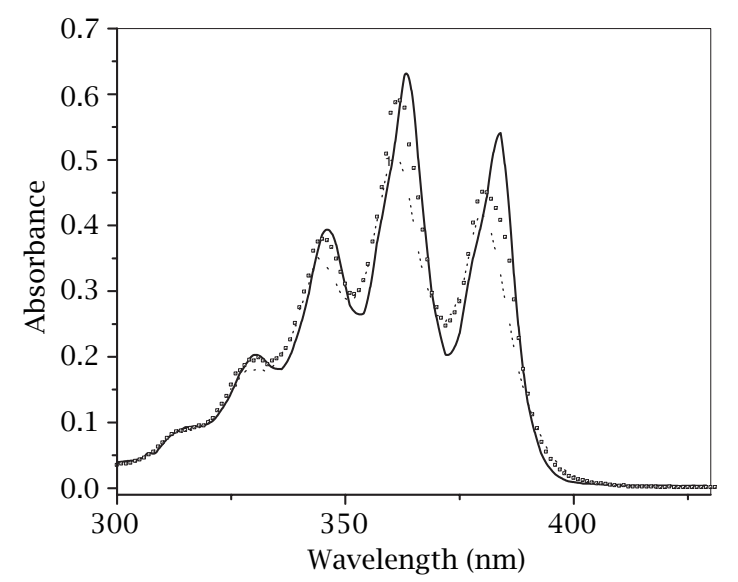

Figure 1. Absorption spectra of $2 \times 10^{-5} \mathrm{M}$ solutions of ANCA in $\mathrm{H}_{2} \mathrm{O}$ (-), ethanol (- -) and acetonitrile (....).

In agreement with previously published results, the fluorescence of ANCA exhibits a pronounced solvent and concentration dependence $[1,2,3,4,5,6,7]$. In water, the fluorescence spectrum is sharp and structured similar to the unsubstituted anthracene. No practical change in the shape of the structured fluorescence band was observed by increasing the concentration of the ANCA up to $10^{-4} \mathrm{M}$ (See Figure 3). In ethanol solution of $10^{-5} \mathrm{M}$ ACNA however, a peculiar broad fluorescence band with a maximum around
$470 \mathrm{~nm}$ is observed which is partially overlapped with the structured spectrum. The long wavelength broad band prevails in aprotic solvents. This has been explained previously on the bases of different controversial concepts; acid-base equilibrium [3, 4], monomerdimer equilibrium [2], dimer-tetramer equilibrium [5].

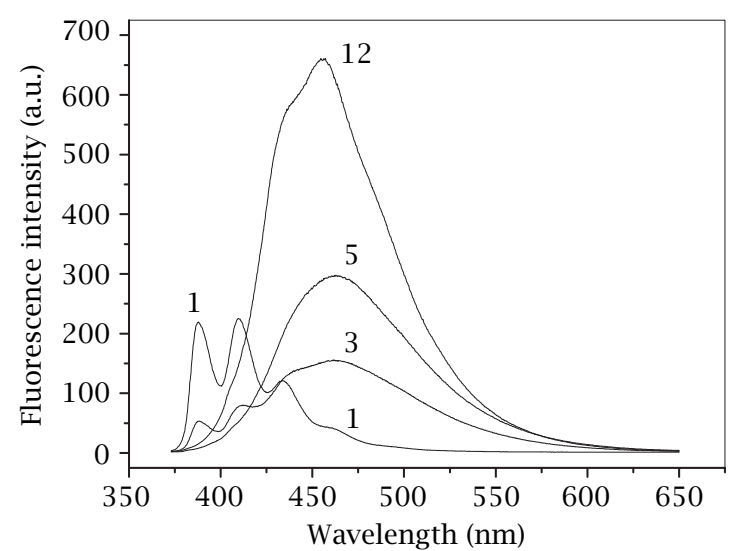

Figure 2. The fluorescence spectra of $2.5 \times 10^{-6} M$ solutions of ANCA in different solvents (the numbers are referred to solvents in Table 1) obtained by excitation at $363 \mathrm{~nm}$.

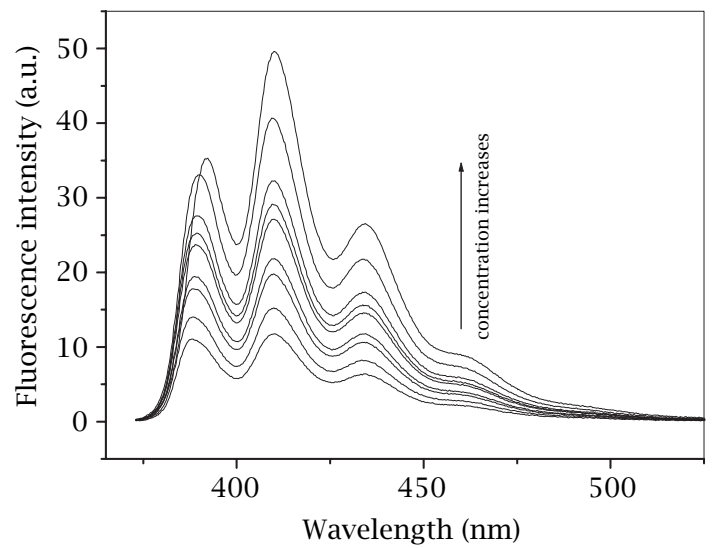

Figure 3. The effect of increasing concentration of ANCA in aqueous solutions on the fluorescence spectrum $(363 \mathrm{~nm}$ excitation wavelength) in the range from $2 \times 10^{-6} \mathrm{M}$ up to $2.5 \times 10^{-5}$.

The observed tail around $400 \mathrm{~nm}$ in the absorption spectrum of ANCA in aprotic solvents (Figure 1) was also observed in micellar solution of ANCA and was attributed by Momiji et al. to a ground state dimeric complex formed in the hydrophobic micellar core [2]. These results point to ground state intermolecular hydrogen bonding formation between carboxyl groups of the ANCA that is prevailing in aprotic solvents like cyclohexane and acetonitrile. Detailed analysis of solvent effect is discussed below.

Figure 2 shows the fluorescence spectra of $2.0 \times$ $10^{-6} \mathrm{M}$ ANCA in various protic and aprotic solvents. In water and alcohols, the observed fluorescence spectrum is more sharp and structured. This is expected since solvation and hydrogen bonding of the solvent molecules with $\mathrm{COO}^{-}$and $\mathrm{COOH}$ groups should inhibit the free rotation of these groups in both ground 
and excited states, which sharpens the fluorescence spectra [1].

On the other hand, broad fluorescence around
$470 \mathrm{~nm}$ is observed in almost every aprotic solvent concomitant with gradual increase of intensity as the solvent polarity decreases. The same broad fluorescence,

Table 1. The effect of solvents on the spectroscopic properties of ANCA $\left(2.0 \times 10^{-6} \mathrm{M}\right)$.

\begin{tabular}{|c|c|c|c|c|c|c|c|}
\hline & solvent $^{\dagger}$ & $\alpha$ & $\beta$ & $\pi$ & $\lambda_{\mathrm{a}}(\log \varepsilon)^{*}$ & $v_{\mathrm{em}}^{\max }\left(\mathrm{cm}^{-1}\right)$ & $v_{\mathrm{a}}-v_{\mathrm{em}}\left(\mathrm{cm}^{-1}\right)$ \\
\hline 1 & $\mathrm{H}_{2} \mathrm{O}$ & 1.17 & 0.18 & 1.09 & $\begin{array}{l}333(4.47) \\
345(4.455) \\
363(4.66) \\
383(4.44)\end{array}$ & 24448 & 3100 \\
\hline 2 & methanol & 0.93 & 0.62 & 0.6 & $\begin{array}{l}329(4.48) \\
346(4.74) \\
362(4.87) \\
383(4.44)\end{array}$ & 24446 & 3177 \\
\hline 3 & ethanol & 0.83 & 0.77 & 0.54 & $\begin{array}{l}330(4.46) \\
346(4.77) \\
363(4.87) \\
383(4.44)\end{array}$ & 24354 & 3194 \\
\hline 4 & isopropyl alc & 0.76 & 0.95 & 0.76 & $\begin{array}{l}332(4.04) \\
347(4.31) \\
364(4.48) \\
383(4.43)\end{array}$ & 22694 & 4777 \\
\hline 5 & acetonitrile & 0.19 & 0.31 & 0.75 & $\begin{array}{l}328(4.49) \\
344(4.55) \\
361(4.63) \\
381(4.44)\end{array}$ & 22694 & 5006 \\
\hline 6 & DMSO & 0 & 0.76 & 1 & $\begin{array}{l}345(4.48) \\
372(4.57) \\
393(4.42)\end{array}$ & 21946 & 4935 \\
\hline 7 & DMF & 0 & 69 & 0.88 & $\begin{array}{l}322(4.40) \\
348(4.60) \\
365(4.73) \\
383(4.43)\end{array}$ & 21984 & 5412 \\
\hline 8 & THF & 0 & 0.52 & 0.57 & $\begin{array}{l}329(3.30) \\
345(4.59) \\
361(4.78) \\
381(4.44)\end{array}$ & 21984 & 5716 \\
\hline 9 & acetone & 0.08 & 0.48 & 0.71 & $\begin{array}{l}345(4.26) \\
361(5.49) \\
380(4.44)\end{array}$ & 21870 & 5830 \\
\hline$\overline{10}$ & $\mathrm{CH}_{2} \mathrm{Cl}_{2}$ & 0 & 0 & 0.82 & $\begin{array}{l}331(4.39) \\
347(4.61) \\
363(4.77) \\
383(4.44)\end{array}$ & 21318 & 6229 \\
\hline 11 & ethylacetate & 0 & 0.45 & 0.55 & $\begin{array}{l}328(3.39) \\
344(4.03) \\
361(4.27) \\
380(4.44)\end{array}$ & 21984 & 5716 \\
\hline$\overline{12}$ & cyclohexane & 0 & 0 & 0 & $\begin{array}{l}328(3.39) \\
344(4.03) \\
361(4.27) \\
380(4.44)\end{array}$ & 22138 & 5562 \\
\hline 13 & n-heptane & 0 & 0 & -0.08 & $\begin{array}{l}325(4.45) \\
344(4.50) \\
360(4.58) \\
379(4.44)\end{array}$ & 22023 & 5754 \\
\hline
\end{tabular}

${ }^{\dagger}$ See reference [12] and the text for the meanings of the solvent parameters $\alpha, \beta$ and $\pi$.

$* \lambda_{\mathrm{a}}$ is the maximum absorption wavelength in (nm), $\varepsilon=$ molar absorptivity in $\left(\mathrm{l} \cdot \mathrm{mole} \mathrm{e}^{-1} \cdot \mathrm{cm}^{-1}\right)$. 
though weaker, was observed in ethanol and SDS micellar solutions [1, 2, 3, 4, 5, 6]. The origin of this broad fluorescence is obscure, even though various groups as overviewed in the introduction of this article exerted considerable explanation efforts. A suggestion that goes along with the common sense is that neutral ANCA molecules should be hardly soluble in hydrocarbons due to the hydrophilic carboxyl group. Hydrogen bonded dimer are expected to have a dissolving ability in the same solvent because the hydrophilic character of the carboxyl group is masked by intermolecular hydrogen bonding. ANCA is thus dissolving as anions in water (and to some extent in alcohols), but it tends to aggregate into hydrogen-bonded dimers (or even cluster) in hydrophobic solvents and in the hydrophobic micellar cores. The excited state enables the rotation of the two-carboxyl groups into the plane of anthracene rings, which brings the broad fluorescence into effect [3]. This suggestion is also supported by the report of Swayambunathan and Lim [14] since they found that ANCA exists mainly in the hydrogen-bonded dimer form in supersonic free jets and that electronic excitation leads to rotation of the dimer bridge of the two carboxyl groups into the plane of the anthracene rings.

An important observation remains to be discussed, namely the fluorescence shoulder around $440 \mathrm{~nm}$ appears in aprotic nonpolar solvents, e.g., THF, cyclohexane, and n-heptane. This shoulder is not observed in the aprotic polar solvent acetonitrile and dimethylsulfoxide. Momiji et al. [2] resolved the fluorescence spectrum of the neutral ANCA as intercalated in dimethyl dioctadecylammonium-montmorillonite at law loading level. The fluorescence spectrum showed maximum emission around $445 \mathrm{~nm}$ [2]. We thus assign the shoulder observed around $440 \mathrm{~nm}$ to the neutral ANCA; (AN COOH) as shown in Scheme 1.

Apparently, ethanol provides an environment in which all species are existing together. Inspection of the fluorescence spectrum of ANCA in ethanol in Figure 2 reveals this fact clearly. Peaks around 388 and $412 \mathrm{~nm}$ belongs to the anion $\mathrm{AN} \mathrm{COO}^{-}$, shoulder around $440 \mathrm{~nm}$ is attributed to the neutral acid ANCA, and the broad band around $470 \mathrm{~nm}$ is due to the hydrogen-bonded dimer form. The broad band is concentration dependent as shown in Figure 4 where the fluorescence spectrum of the hydrogen-bonded dimer is enhanced effectively with increasing the ANCA concentration. This spectrum was obtained by subtracting the spectrum of $1 \times 10^{-6} \mathrm{M}$ solution of ANCA in pure ethanol from the spectra at higher concentrations.

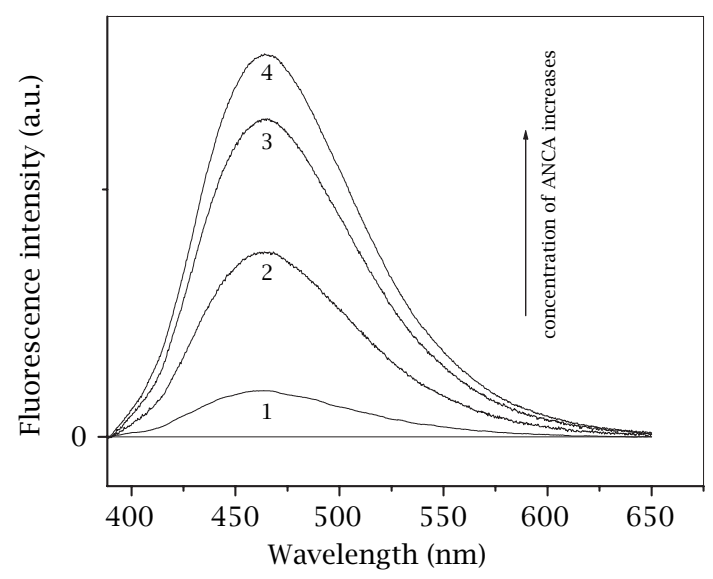

Figure 4. The effect of concentration of ANCA in ethanol solution: (1) $2 \times 10^{-6} \mathrm{M}$, (2) $1.4 \times 10^{-5} \mathrm{M}$, (3) $2.1 \times 10^{-5} \mathrm{M}$ and (4) $2.5 \times 10^{-5} \mathrm{M}$ on the net fluorescence spectrum $\mathrm{ob}$ tained by subtracting the fluorescence of $1 \times 10^{-6} \mathrm{M} \mathrm{ANCA}$ in ethanol from each obtained spectrum at higher concentrations.

The overall processes and species discussed above are depicted in Scheme 1, which expresses a hypothetical general case regardless the type of solvent.

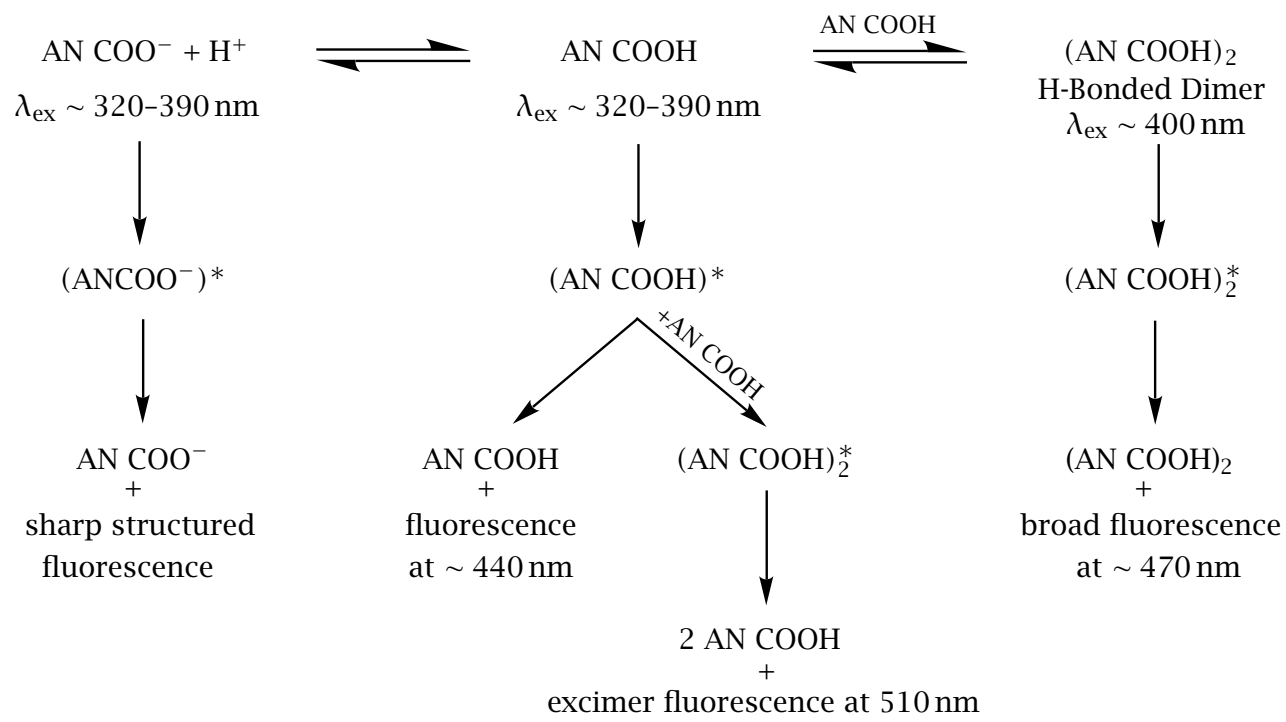

Scheme 1. (AN = Anthracene moiety). 
It should be noted that excimer fluorescence depicted in Scheme 1 was reported at $510 \mathrm{~nm}$ in the cases of ANCA crystals [8] and ANCA intercalated in dimethyl dioctadecyl ammonium-montmorillonite at very high loading levels [2]. This excimer is not observed in our case because the excited lifetime of ANCA ( few ns) does not allow the encounter of the excited state with another ANCA molecule under the present condition of very low concentration. The fluorescence decay curve of ANCA in ethanol at $\lambda_{\mathrm{f}}=357 \mathrm{~nm}$ could be fitted monoexponentially; $\tau_{\mathrm{f}}^{357}$ (ethanol) $=3.66 n s$, whereas that at $468 \mathrm{~nm}$ was fitted biexponentially with a slower component of $\tau_{\mathrm{f}}^{468}=9.2 \mathrm{~ns}$. The slower component is clearly due to the excited state of the hydrogen-bonded dimer.

From our experimental observations the two equilibria depicted in Scheme 1 are strongly affected by the concentration and the type of solvent. For instance, in water the equilibrium is shifted towards the $\mathrm{ACOO}^{-}$ whereas increasing the concentration would naturally shift the equilibrium to the dimer formation. Dimer formation is also enhanced in aprotic solvents as explained above.

The above mentioned findings prompted us to study the effect of the interplay between various solvent properties on the fluorescence emission in the binary acetone/ethanol solvent mixture. Interestingly, the broad band fluorescence was "quenched" upon increasing the concentration of alcohol in acetone as shown in Figure 5. Direct quenching through Stern-Volmer mechanism is not operating in the present case because the smallest concentration employed of ethanol is about $10^{6}$ times as large as ANCA concentration. Alternatively one may think of a mechanism involving the preferential solvation of the ANCA in micro-aggregates of ethanol clusters in acetone solvent. The formation of ACNA dimer is then less favorable due to hydrogen-bonding solvation by alcohol molecules that keep ACNA molecules in the monomer form. This is direct evidence supporting the mechanism of ground state dimer formation.

The idea of preferential solvation due to microheterogeneity in polar-nonpolar binary solvent mixture is well established [9, 10, 11]. The microheterogencity in the case of acetone-ethanol mixture is intuitively due to the formation of hydrogen bonded clusters of ethanol molecules which are able to accommodate ACNA molecules. The fluorescence of ANCA molecules (or dimers) would then sensitively probe the nature of the medium. This suggestion explains why in pure acetone only the broad fluorescence from the dimer is observed, whereas gradual increase of ethanol led eventually to the observation of the fluorescence of (ANCA $+\mathrm{AN}^{-} \mathrm{COO}^{-}$).

The above discussion clearly demonstrates how sensitive is the ANCA to the nature of the solvent. To find out the relative role of the various solvent properties such as hydrogen-bond donor acidity $\operatorname{HBD}(\alpha)$, the hydrogen-bond acceptor (HBA) behavior $(\beta)$ and the intrinsic polarity $(\pi)$ related to the dipole mo- ment of the solvent, we have treated our data using the multiparameter equation of Kamlet, Abboud, Abraham and Taft [9, 12]. When statistical analysis was applied to the spectral data given in Table 1 and employing the parametric values of the solvents in Table 1 , the Stockes' shift, $\Delta v$ in $\mathrm{cm}^{-1}$, fits into the following multilinear regression function $\Delta v=\Delta v_{0}+\mathrm{a} \alpha+\mathrm{b} \beta+$ $\mathrm{c} \pi$ (Where $\Delta v_{0}=5798, \mathrm{a}=-2462, \mathrm{~b}=-1024$ and $\mathrm{c}=393$ with excellent correlation coefficient $\left(\mathrm{r}^{2}\right)$ of 0.954). Thus, it is clear from the obtained coefficients that H-bonding donor-acceptor properties of the solvents dominate the overall solvent effect in this case.
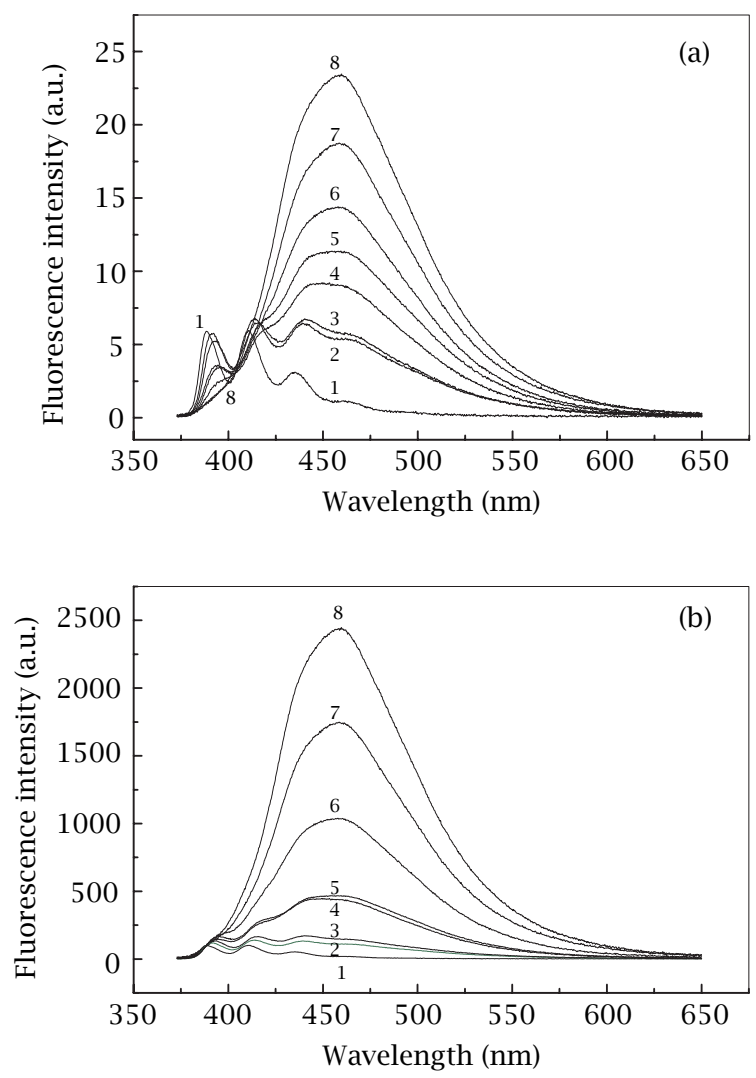

Figure 5. Fluorescence spectra of ANCA $\left(1.0 \times 10^{-6} M\right)$. (a) In different ethanol/acetone mixtures ( $v / v)$ : (1) 100\% ethanol, (2) $50 \%$, (3) $40 \%$, (4) $35 \%$, (5) $30 \%$, (6) $15 \%$, (7) $10 \%$ and (8) $0 \%$ (100\% acetone). (b) And normlized at $388 \mathrm{~nm}$.

Trials made to fit other spectroscopic data using similar multilinear equations were unsuccessful due to the complex nature of the effect of the solvent, on the overlapped various ACNA molecular species in the spectral region concerned.

3.2. Photophysics of ANCA in CTAB micelles. The fluorescence spectra of ANCA were also studied in surfactant solutions of CTAB as a function of surfactant concentration. In addition to an anthracene-like sharp fluorescence, the broad fluorescence with a peak around $470 \mathrm{~nm}$ also appeared at $330 \mathrm{~nm}$ excitation. Similar results were cited for sodium dodecyl sulfate and Triton $\mathrm{X}-100$. Only a broad fluorescence was seen at $400 \mathrm{~nm}$ excitation. Based on the excitation and absorption spec- 
tra, it has been suggested that the broad fluorescence is due to emission of the hydrogen-bonded dimers dissolved in the micelles [2]. The fluorescence spectra of ANCA showed a broad peak with increase in loading levels of ANCA in the CTAB (see Figure 6). The fluorescence was not similar to the excimer in a head-to-head configuration with a maximum at $510 \mathrm{~nm}$ observed in the crystals.

Therefore, the hydrogen-bonded dimers are the origin of the broad fluorescence in the interlayer spaces of micelles.

The broad band around $470 \mathrm{~nm}$ is clearly observed when the concentration of ANCA was $10^{-3} \mathrm{M}$ (see Figure 6). Due to the hydrophobicity of the anthracene moiety, ANCA molecules are expected to direct the $\mathrm{COO}^{-}$and $\mathrm{COOH}$ polar groups into the surface near the cationic end of the micelles at water-micelle interface with the anthracene part impeded to the micellar core. Under such circumstances high occupation numbers are expected and the H-bonding dimer formation becomes highly probable at large ANCA concentration.

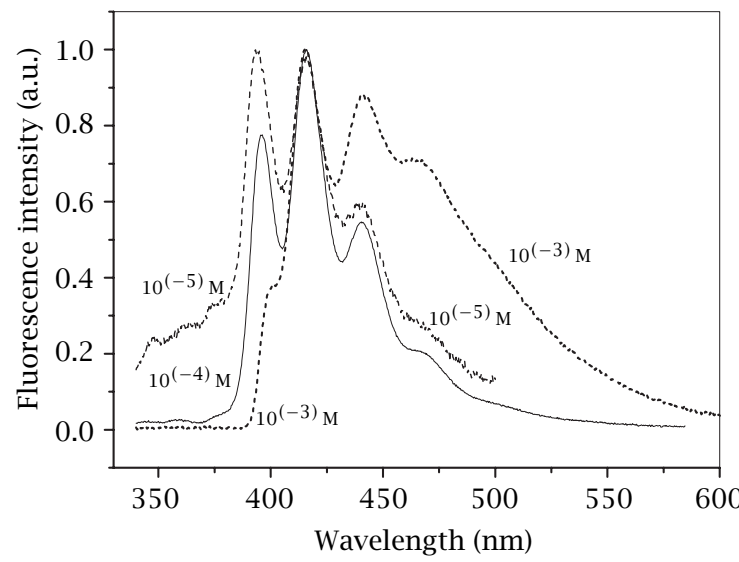

Figure 6. The fluorescence spectra of different concentrations of ANCA $\left[(-) 10^{-5} \mathrm{M},\left(-\right.\right.$ - $^{-} 10^{-4} \mathrm{M}$ and (....) $\left.10^{-3} \mathrm{M}\right]$ in aqueous $C T A B$ solutions $(0.4 \mathrm{M}$, above the critical micelle concentration of CTAB of about $10^{-3} \mathrm{M}$ ) .

3.3. Photostability studies. The ANCA was found to be photolabile in aqueous media of different pHs. The acid-base catalyzed photodegradation rate was studied by following up absorption and/or fluorescence intensi- ties as a function of illumination dose (Figure 7). The determined rate of photochemical degradation of ANCA depends remarkably on the nature of the medium as seen in Figure 8. The pseudo first order degradation rate constant is remarkably enhanced in heterogeneous medium of CTAB due to the easily formed (identified in the GC-Mass spectrum) photolabile head-to-head dimer of ANCA formed by irradiation with light (Figure 9).

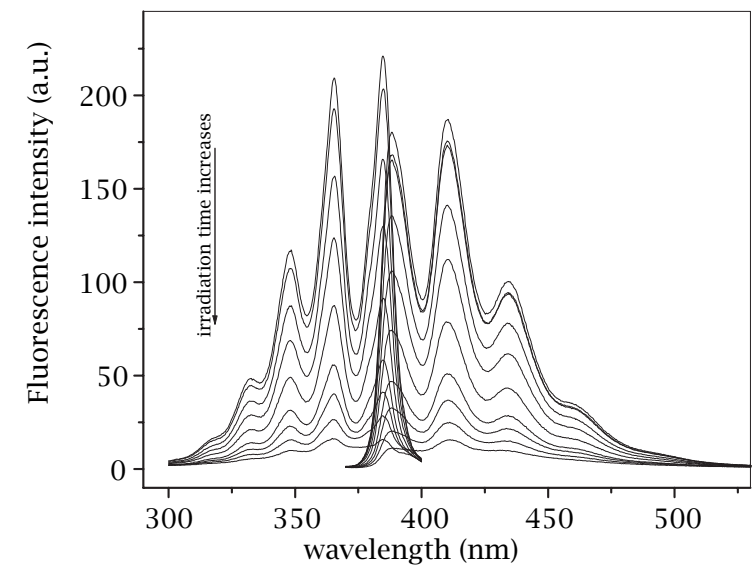

Figure 7. The decrease in fluorescence and excitation spectra of ANCA solution of $\mathrm{pH} 6$ as a function of increasing time of irradiation (at $20^{\circ} \mathrm{C}$ ). Similar plots were obtained in other media and at different temperatures (see the text) with different rates of degradation.

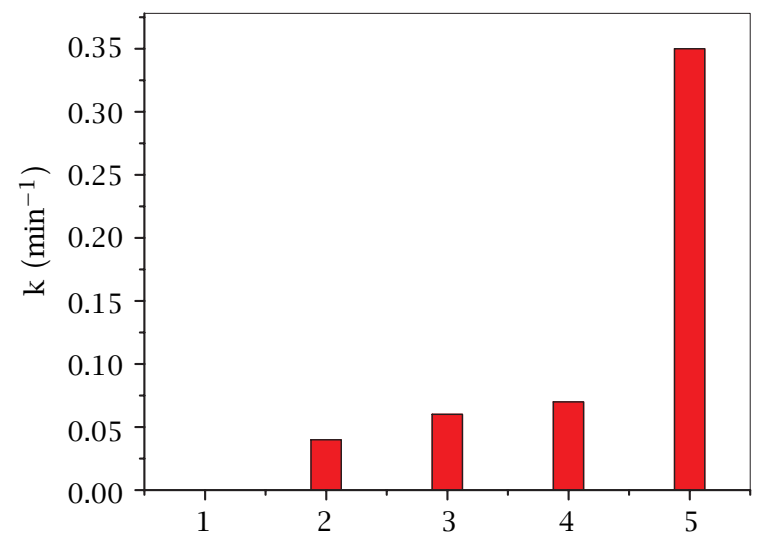

Figure 8. The effect of the medium on the rate constant of photodegradation of ANCA: (1) ANCA in ethanol, (2) $\mathrm{H}_{2} \mathrm{O}$ solution, (3) $\mathrm{pH} 12.0$ (4) $\mathrm{pH} 6.0$ and (5) CTAB.
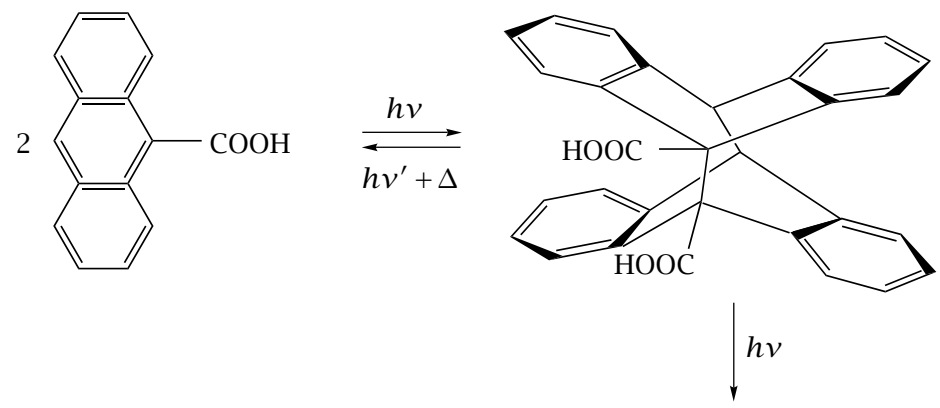

Photooxidation Products

Figure 9. The effect of light irradiation on ANCA degradation via head-to-head dimer formation. 
Consequently, further photodegradation processes are initiated. As expected, the determined activation energy of Photodegradation is low $\left(\sim 3.2 \mathrm{~kJ}\right.$. mol $\left.{ }^{-1}\right)$. This result favors photooxidation process. Anthraquinone was the main photodegradation product identified by GC-Mass technique. Our results are in agreement with the recently published findings on the Photodegradation and modification of some toxic polycyclic aromatic hydrocarbons including anthracene [15].

\section{CONCLUSION}

Formation of the hydrogen-bonded associated dimers of ANCA in the ground state is evident from the absorption spectra in different media. The dimer formation is favored in aprotic solvents and micellar environments. Nature of the medium and concentration of ANCA are very important factors that affect the acid-base and monomer-dimer equilibria in the excited state. Analysis of the solvent effects on the value of Stokes' shift revealed that H-bonding donor-acceptor properties of the solvents dominate the overall solutesolvent interactions that are more pronounced in the excited state of ANCA.

Photolability of ANCA in aqueous media of different pHs and in CTAB solutions is due to the acid-base catalyzed photodegradation of the anionic ANCA. Photolability is more pronounced in micellar solutions of $\mathrm{CTAB}$ due to the initial formation of thermally and photochemically labile photodimer, which favors further degradation via photooxidation process.

\section{REFERENCES}

[1] T. C. Werner and D. M. Hercules, J. Phys. Chem. 73 (1969), 2005.

[2] I. Momiji, C. Yoza, and K. Matsui, J. Phys. Chem. B. 104 (2000), 1552 (and references cited therein).

[3] S. Suzuki, T. Fujii, N. Yoshiike, S. Komatsu, and T. Iida, Bull. Chem. Soc. Jpn. 51 (1978), 2460.

[4] N. S. Bazilevskaya and A. S. Cherkasov, Opt. Spectrosc. 18 (1965), 30.

[5] N. Ghoneim, D. Scherrer, and P. J. Suppan, Lumin. 55 (1993), 271.
[6] J. Froemmel and T. Wolff, J. Colloid Interface Sci. 201 (1998), 86.

[7] A. F. M. Dessouky, M. El-Naggar, H. R. Galal, D. Mekkawi, G. M. Attya, and M. S. A. Abdel-Mottaleb, The 5th international Conference on Solar Energy Storage and Applied Photochemistry (SOLAR'99), April, 1999, Cairo, Egypt.

M. S. A. Abdel-Mottaleb and G. M. Attya, American Society for Photobiology 27th Annual Meeting, July 1999, Washington, DC, USA.

David R. Worrall, Sian L. Williams, and Francis Wilkinson, J. Phys. Chem. B 101 (1997), 4709. Joykrishna Dey and Isiah M. Warner, J. Phys. Chem. A 101 (1997), 4872.

T. Hirsch, H. Port, H. C. Wolf, B. Miehlich, and F. Effenberger, J. Phys. Chem. B 101 (1997), 4525. Ignacio Martini, Jose H. Hodak, and Gregory V. Hartland, J. Phys. Chem. B 103 (1999), 9104.

Ignacio Martini, Jose H. Hodak, and Gregory V. Hartland, J. Phys. Chem. B 102 (1998), 9508.

Shuichi Hashimoto, Sigeru Ikuta, Tsuyoshi Asahi, and Hiroshi Masuhara, Langmuir 15 (1998), 4284. Hiroyuki Yoshikawa, Keiji Sasaki, and Hiroshi Masuhara, J. Phys. Chem. B 104 (2000), 3429.

G. Gery, H. Fukumura, and H. Masuhara, J. Phys. Chem. B 101 (1997), 3698.

[8] M. D. Cohen, Z. Ludmer, and V. Yakhot, Phys. Status Solidi B. 67 (1975), 51.

[9] C. Reichardt, Solvent Effect in Organic Chemistry, Verlag Chemie, Weinheim, 1979, ch. 2.

[10] N. Kh. Petrov, A. Wiessner, T. Fiebig, and H. Staerk, Chem. Phys. Lett. 241 (1995), 172.

[11] N. Kh. Petrov, J. Phys. Chem. A 102 (1998), 7878.

[12] M. J. Kamlet, J. M. Abboud, M. H. Abraham, and R. W. Taft, J. Org. Chem. 48 (1983), 2877.

[13] T. Wolff and N. Muller, J. Photochem. 23 (1983), 131.

[14] V. Swayambunathan and E. C. Lim, J. Phys. Chem. 91 (1987), 6359.

[15] A. Mallakin, D. G. Dixon, and B. M. Greenberger, Chemosphere 40 (2000), 1435. 


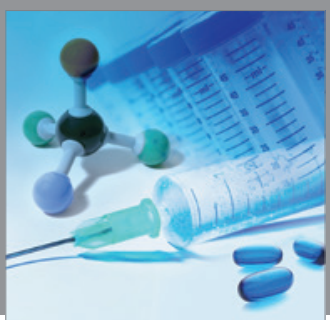

International Journal of

Medicinal Chemistry

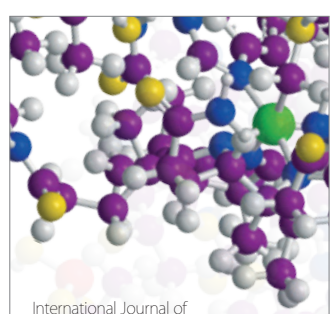

Carbohydrate Chemistry

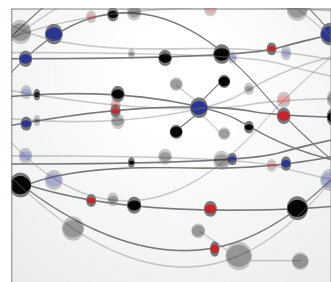

The Scientific World Journal
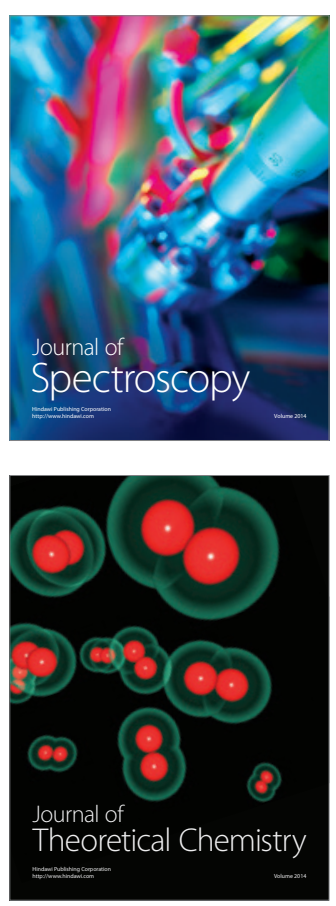
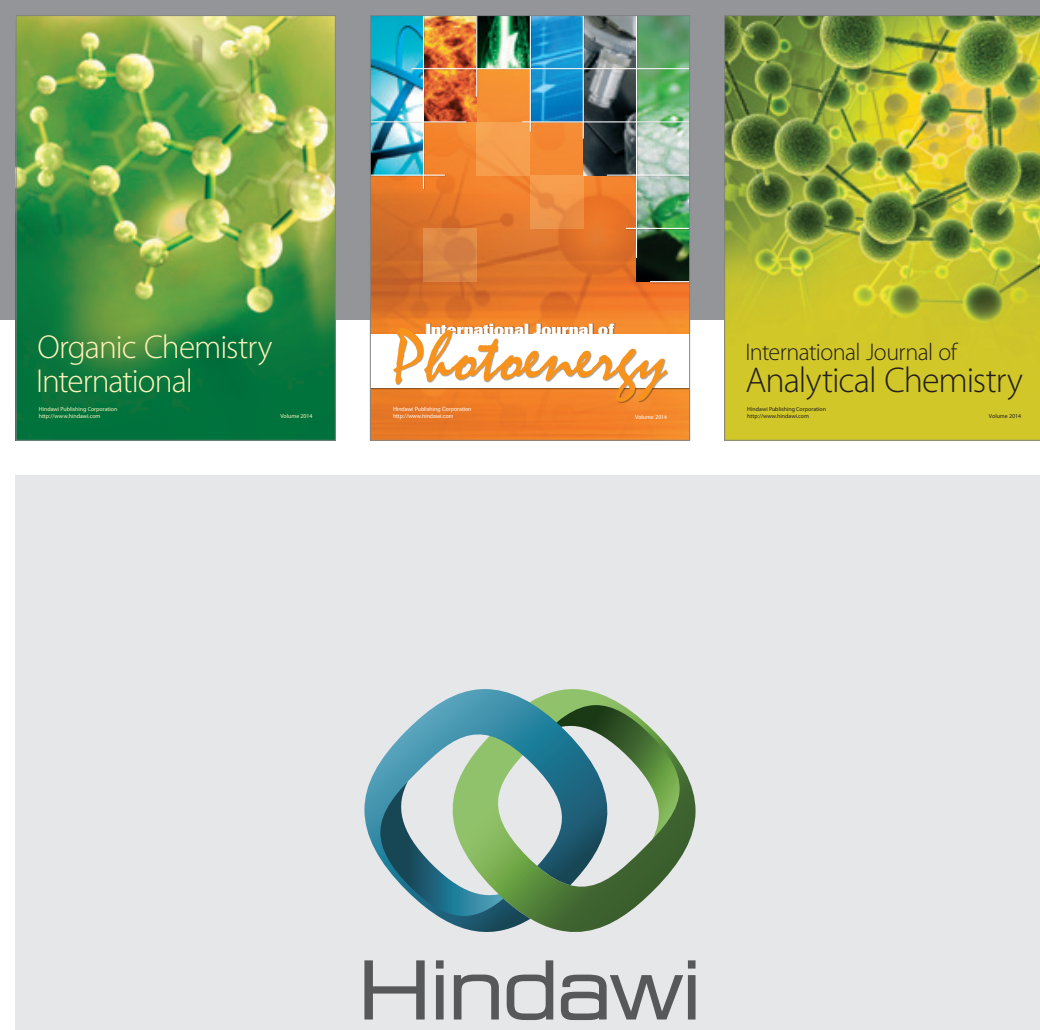

Submit your manuscripts at

http://www.hindawi.com
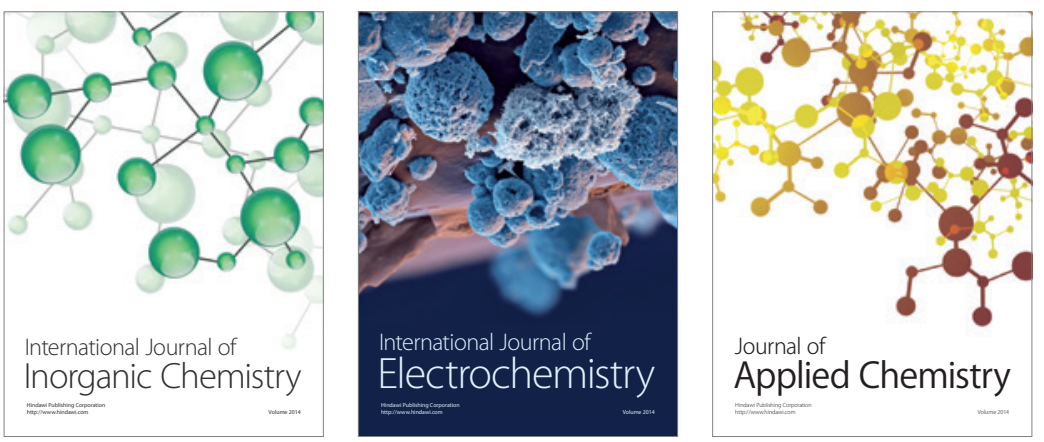

Journal of

Applied Chemistry
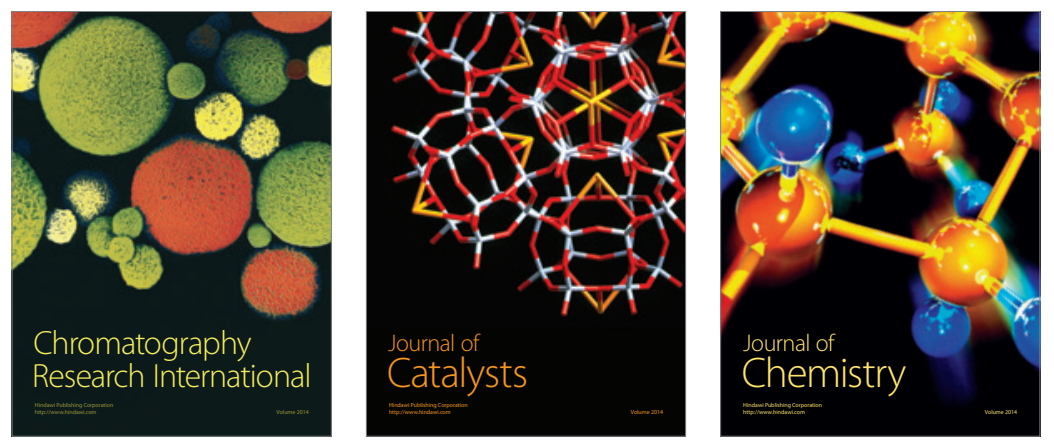
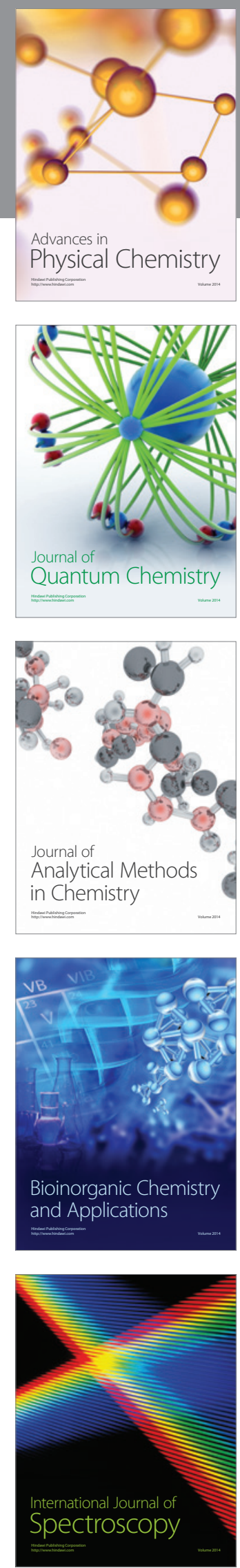